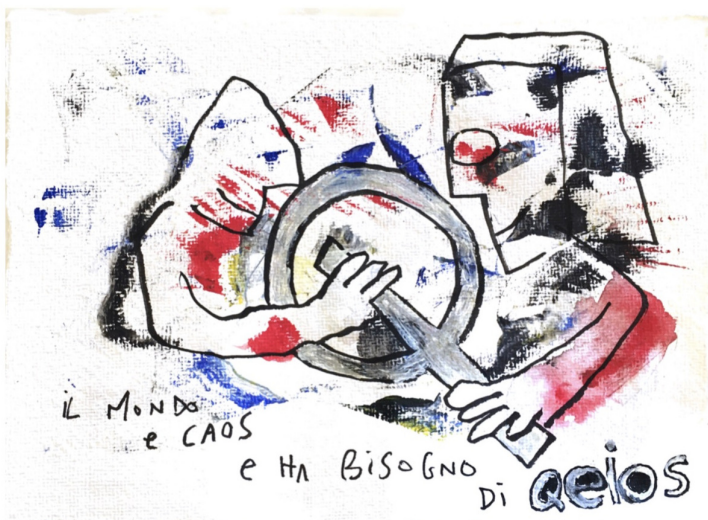

\title{
Curative Rates of Medication-Related Osteonecrosis of the Jaw Following Application of the SICMF-SIPMO Surgical Treatment Algorithm
}

\author{
Giordana Bettini ${ }^{1}$, Giorgia Saia ${ }^{2}$, Elena Sofia Perra ${ }^{2}$, Laura Tognin ${ }^{3}$, Piero Franco $^{2}$, Giorgio Bedogni ${ }^{4}$, Alberto Bedogni $^{2}$ \\ 1 University-Hospital of Padova \\ 2 University of Padua \\ 3 University of Parma \\ 4 University of Bologna
}

Funding: The author(s) received no specific funding for this work.

Potential competing interests: A.B. received honoraria from Amgen. The remaining authors declared that no potential competing interests exist.

\section{Abstract}

Background. Management of Medication related osteonecrosis of the jaw (MRONJ) is challenging and there is little evidence about the effectiveness of treatments.

The present study aims at assessing the value of the SICMF-SIPMO MRONJ staging system as a predictor of treatment success.

Patients \& Methods. We performed a 10-years longitudinal cohort study at the Unit of Maxillofacial Surgery of Padova University (Italy). The study was approved by the local Ethical Committee of the University Hospital of Padova (CESC 4920/AO/20 - 24 September 2020). Patients were included in the study if they satisfied the SICMF-SIPMO clinicoradiological diagnostic criteria of $\mathrm{MRONJ}$ and were staged accordingly. Patients were assigned to each surgical treatment based on the SICMF-SIPMO classification. Patients were followed up at three-month intervals up to 1-year and underwent a CT scan of the jaws at three, six, and twelve months postoperatively. 
Results. Overall, a total of 70 patients reached the 12-month follow-up and 75 operated jaw sites were available for the analysis. The cumulative curative rate (CR) at 1-year follow-up was $85.3 \%$. MRONJ recurred in 9 jaw sites (12\%). The curative rates did not significantly differ between maxilla and mandible ( $87 \%$ vs. $84 \%)$. The underlying disease highly influenced the $\mathrm{CR}$ of $\mathrm{MRONJ}$, with cancer patients more likely to develop recurrences within 1 year (CR cancer $=76 \%$; CR osteoporosis $=100 \%)$. Bone curettage and sequestrectomy showed the highest CR in MRONJ stage 1, regardless of the underlying disease (88.2\%). Marginal resection proved to be successful in MRONJ stage 2 with an overall curative rate of $81 \%$. Nevertheless, MRONJ stage 2 patients with cancer showed high recurrence rates when treated with marginal resection. Segmental resection proved highly successful in MRONJ stage 2 (88.2\%) and MRONJ stage 3 (92.9\%). Of note, segmental resections in MRONJ stage 2 were mostly performed in cancer patients (12/15).

Conclusion. In conclusion, the stage-related surgical algorithm proposed by SICMF-SIPMO can be safely used and it should be implemented to select the appropriate surgical treatment for MRONJ patients. Further studies are warranted before its final validation.

\section{Definitions}

Medication related osteonecrosis of the jaw (MRONJ)

Defined by Alberto Bedogni et al.

\section{Effectiveness}

Defined by National Cancer Institute

Clinical Trial Eligibility Criteria

Defined by National Cancer Institute

Spreadsheet

Defined by National Cancer Institute

Consecutive

Defined by National Cancer Institute

Retrospective Study

Defined by National Cancer Institute

\section{Introduction}

\section{Medication related osteonecrosis of the jaw}

Management of (MRONJ)

is challenging and there is little evidence about the effectiveness of treatments.

Treatment strategies of MRONJ have been originally developed based on a clinical staging system endorsed by the American Association of Oral and Maxillofacial Surgery (AAOMS). ${ }^{[1]}$ Patients with mild (stage 1) to moderate (stage 2) disease are offered non-invasive treatments such as control of infection and pain, and superficial debridement of bone, whereas only those patients with advanced and refractory disease (stage 3) may benefit from surgery. This staging system has been later modified to be more inclusive, ${ }^{[2]}$ but no attempt to revise treatment strategies has been done at all, 
despite the increasing evidence that surgery might provide long-lasting benefits to MRONJ patients in terms of healing and disease control. [3][4][5]

Over the recent years, the role of imaging has been emphasized as it better displays the real extent of jawbone disease and can integrate the clinical picture of MRONJ. ${ }^{[6]}$ Computer tomography (CT) can detect equally signs of bone necrosis and bone sclerosis, with respect to the uninvolved bone tissue. ${ }^{[7]}$ It can also pick up the early radiological aspects of MRONJ as compared with plain radiographs. ${ }^{[8][9]}$

Since that, the inclusion of CT to measure the extent of jawbone involvement has been advocated not only to improve the diagnostic process of MRONJ but also the patient's assignment to treatments ${ }^{[10]}$.

In 2012, the Italian Societies of Maxillo-facial Surgery (SICMF) and Oral Pathology and Medicine (SIPMO) endorsed a clinico-radiological staging system to assign treatment to MRONJ patients, where surgery was integrated for the first time as a first-line treatment. ${ }^{[11]}$ Surgery was graded based on the real extent of bone disease so that patients with focal disease" (stage 1) are likely to receive less invasive surgical treatment (i.e. bone curettage and sequestrectomy) as compared with more advanced disease stages (stage 2 and 3) who deserve more radical interventions (i.e. marginal and segmental resection of bone). ${ }^{[12][13]}$

Based on the assumption that MRONJ seems to progress more rapidly, and bone necrosis tends to recur more easily in cancer patients than in osteoporosis patients, the SICMF-SIPMO staging system also differentiates the magnitude of surgical treatment based on the underlying diseases (cancer vs. osteoporosis).

As of today, the clinical significance of the SICMF-SIPMO classification system and the prognostic impact of its stagerelated treatment algorithm has not been proved.

The present study aims at assessing the value of the SICMF-SIPMO MRONJ staging system as a predictor of treatment success.

\section{Material and Methods}

\section{Study design and setting}

retrospective cohort

We performed a 10-years study

at the Unit of Maxillofacial Surgery of Padova University (Italy). The study was approved by the local Ethical Committee of the University Hospital of Padova (CESC 4920/AO/20 - 24 September 2020). All subjects gave written informed consent.

\section{Eligibility criteria}

Patients were included in the study if they satisfied the SICMF-SIPMO clinico-radiological diagnostic criteria of MRONJ 
and were staged accordingly. Clinical and radiological data (CT scan) of consecutive patients who underwent surgical treatment of MRONJ between January 2010 and December 2020 were obtained from the prospective database of the Regional Centre for Diagnosis, and Treatment of Medication and Radiation-related Bone Diseases of the Head and Neck (Veneto region). These data were integrated into a computer-based spreadsheet.

Patients were assigned to each surgical treatment based on the SICMF-SIPMO classification. Patients were followed up at three-month intervals up to 1-year and underwent a CT scan of the jaws at three, six, and twelve months postoperatively.

\section{Study outcome}

The primary study outcome was the curative rate (CR) of MRONJ following surgery, which comprises both the SICMFSIPMO definitions of Healing and Remission. ${ }^{[12]}$ Healing was achieved in the case of absent clinical and CT signs of MRONJ at the 12-month follow-up, while Remission was assigned in the case of absent clinical signs of MRONJ and stable radiological CT signs at the 12-month follow-up. Patients who developed clinical and radiological signs of disease within 1 year after surgical treatment were considered Recurrent cases. Patients who displayed disease progression at CT without clinical signs and symptoms were defined as silent MRONJ cases.

The study outcome was then stratified by jaw site (mandible vs. maxilla), primary disease (cancer vs. osteoporosis), treatment type (bone curettage and sequestrectomy vs. marginal resection vs. segmental resection), and SICMF-SIPMO stage (stage 1 vs. stage 2. vs. stage 3), to assess the value of the SICMF-SIPMO MRONJ staging system as a predictor of treatment success.

\section{Statistical analysis}

Descriptive statistics were calculated on a per-patient basis. Continuous variables are reported as median and minimum and maximum values, because of skewed distributions. Categorical variables are given as the number or percentage of patients with the characteristic of interest. Inferential statistics were calculated on a per-bone basis $(n=85)$. Statistical analysis was performed using Stata 16.1 (Stata Corporation, College Station, TX, USA).

\section{Results}

\section{Patient's features}

80 consecutive MRONJ patients underwent surgery according to the SICMF-SIPMO protocol, with a total of 85 operated jaw sites (5 patients received surgery to both maxilla and mandible).

Metastatic breast cancer was the most frequent diagnosis (29\%), followed by primary osteoporosis (27.5\%). Zoledronic acid had been the most used antiresorptive (65\%), followed by alendronate (28\%). (Table 1 ) 


\section{Table 1.}

Baseline features of the population

Male

$23(27 \%)$

Age (years)

$70(65 ;$

77)

European

$80(100 \%)$

Underlying disease

- Breast cancer

$25(29 \%)$

- Multiple myeloma

$10(12 \%)$

- Prostate cancer

$6(7 \%)$

- Lung cancer

$3(4 \%)$

- Melanoma

- Primary osteoporosis

- Secondary osteoporosis

$10(12 \%)$

Comorbidities

- Diabetes mellitus

$10(12 \%)$

- Chronic renal failure

$2(2 \%)$

- Hypertension

$40(47 \%)$

- Cardiovascular disease

- Anemia

- Hypothyroidism

- Arhythmia

$4(5 \%)$

- Psoriasic arthritis

- Rheumatoid arthritis

- Diverticulitis

$1(1 \%)$

- Reactive arthritis

- Hypercholesterolemia

$10(12 \%)$

- Arthrosis

$1(1 \%)$

- COPD

$1(1 \%)$

- Asthma

- Hashimoto disease

- Lymphoproliferative disorder

- HBV

- HCV

- Multiple sclerosis

- Chronic myeloid leukaemia

- Bowen's disease

- Parkinson disease

- Erysipelas 


\begin{tabular}{l|l}
\hline - Sjogren's syndrome & $1(1 \%)$ \\
\hline - Glaucoma & $1(1 \%)$ \\
\hline - Meniere's disease & $1(1 \%)$ \\
\hline - Gastric ulcer & $1(1 \%)$ \\
\hline
\end{tabular}

The most frequent oral trigger of MRONJ development was dental/periodontal infection (56\%), followed by tooth extraction (26\%). At clinical presentation, the most common clinical sign of MRONJ was the presence of a probing bone mucosal fistula ( $80 \%)$, followed by purulent discharge (47\%) and necrotic bone exposure in the oral cavity (44\%). (Table 2)

\begin{tabular}{|c|c|c|}
\hline MRONJ features before surgery & Jaws & $\mathrm{N}(\%)$ \\
\hline MRONJ site & 85 & \\
\hline Maxilla & & $36(42 \%)$ \\
\hline Mandible & & 49 (58\%) \\
\hline SICMF-SIPMO stage at baseline & 85 & \\
\hline - $1 \mathrm{~A}$ & & $17(20 \%)$ \\
\hline - $1 \mathrm{~B}$ & & $5(6 \%)$ \\
\hline - $2 \mathrm{~A}$ & & $17(20 \%)$ \\
\hline - $2 \mathrm{~B}$ & & $29(34 \%)$ \\
\hline - 3 & & $17(20 \%)$ \\
\hline \multicolumn{3}{|l|}{ Precipitating event } \\
\hline - dental or periodontal infection & 85 & $48(56 \%)$ \\
\hline - peri-implant infection & 85 & $5(6 \%)$ \\
\hline - tooth extraction & 85 & $22(26 \%)$ \\
\hline - prosthesis & 85 & $7(8 \%)$ \\
\hline - unknown & 85 & $3(4 \%)$ \\
\hline \multicolumn{3}{|l|}{ Clinical signs at presentation } \\
\hline - Halitosis & 85 & 35 (41\%) \\
\hline . & $\mathrm{xn}^{2}$ & 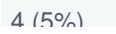 \\
\hline
\end{tabular}




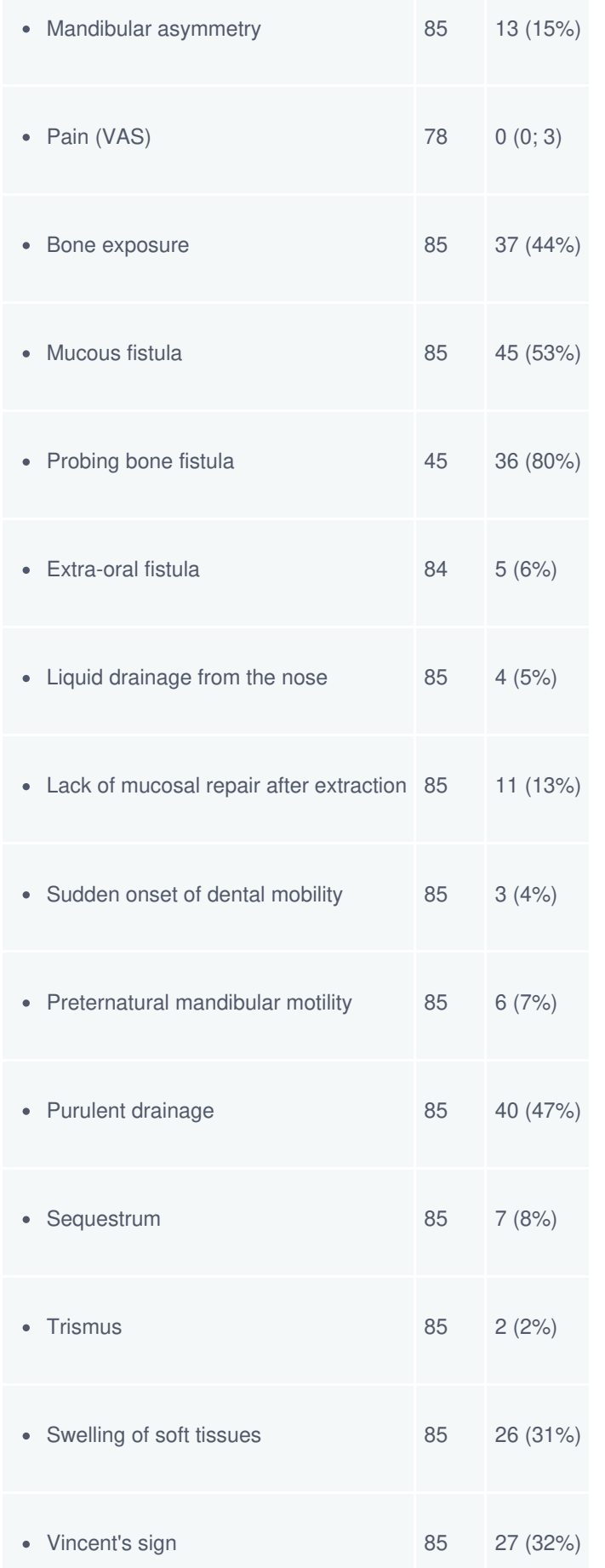

Three patients died and five were lost during the follow-up. Two patients did not conclude the follow-up at present investigation.

\section{Study outcome}

Overall, a total of 70 patients reached the 12-month follow-up and 75 operated jaw sites were available for the analysis. 
The cumulative CR at 1-year follow-up was $85.3 \%$. Of the 64 jaw sites that were cured, $84 \%$ healed completely, while the remaining $17 \%$ displayed remittent disease. MRONJ recurred in 9 jaw sites (12\%), while the remaining two sites displayed silent disease (2.7\%). (Graph 1)

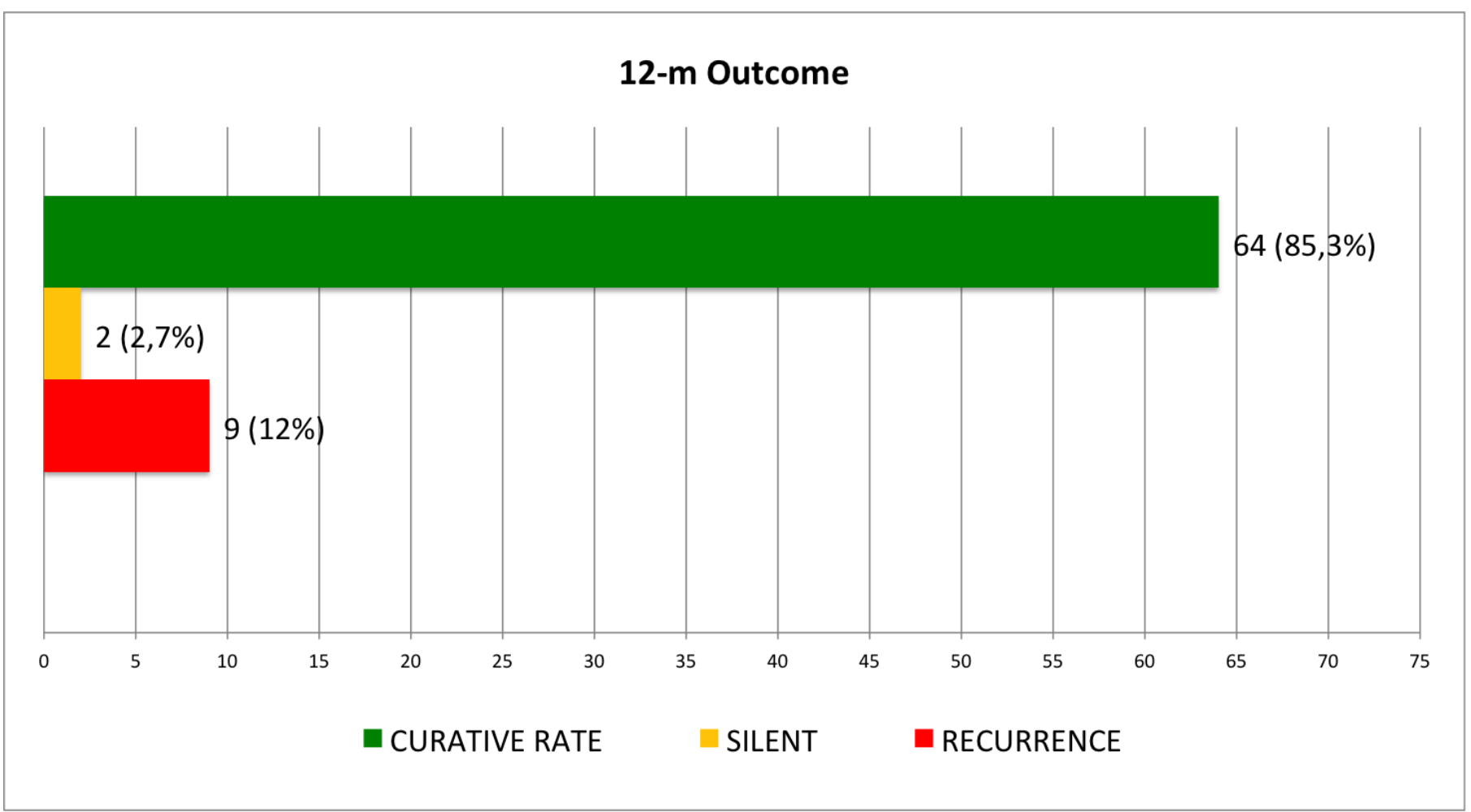

Graph 1.

The result of surgical treatment did not significantly differ between maxilla and mandible. (Graph 2) 


\section{2-m Outcome by site}

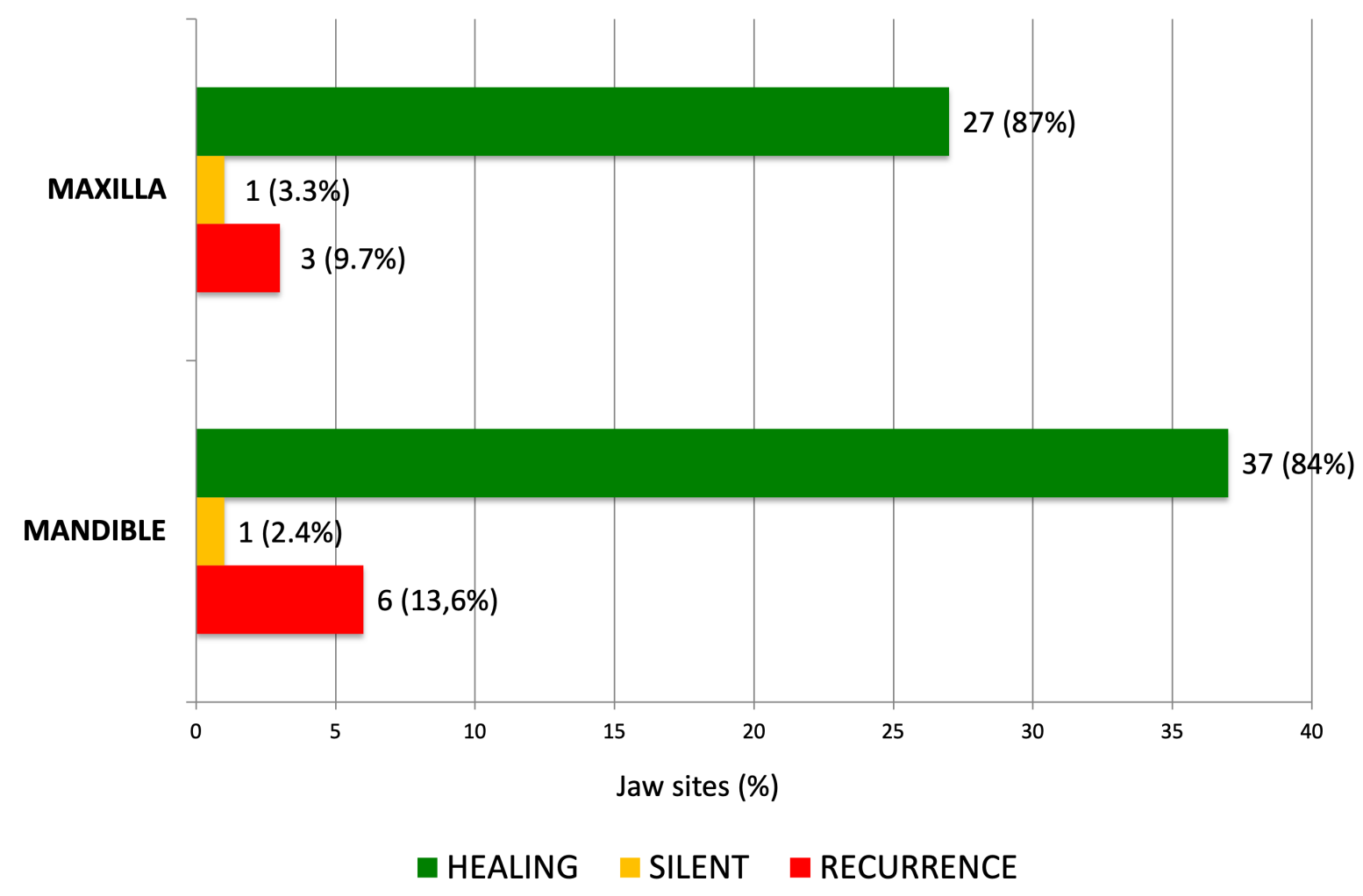

Graph 2.

On the contrary, primary disease influenced the $\mathrm{CR}$ of $\mathrm{MRONJ}$, with cancer patients more likely to develop recurrences within 1 year. (Graph 3) 


\section{2-m Outcome by baseline disease}

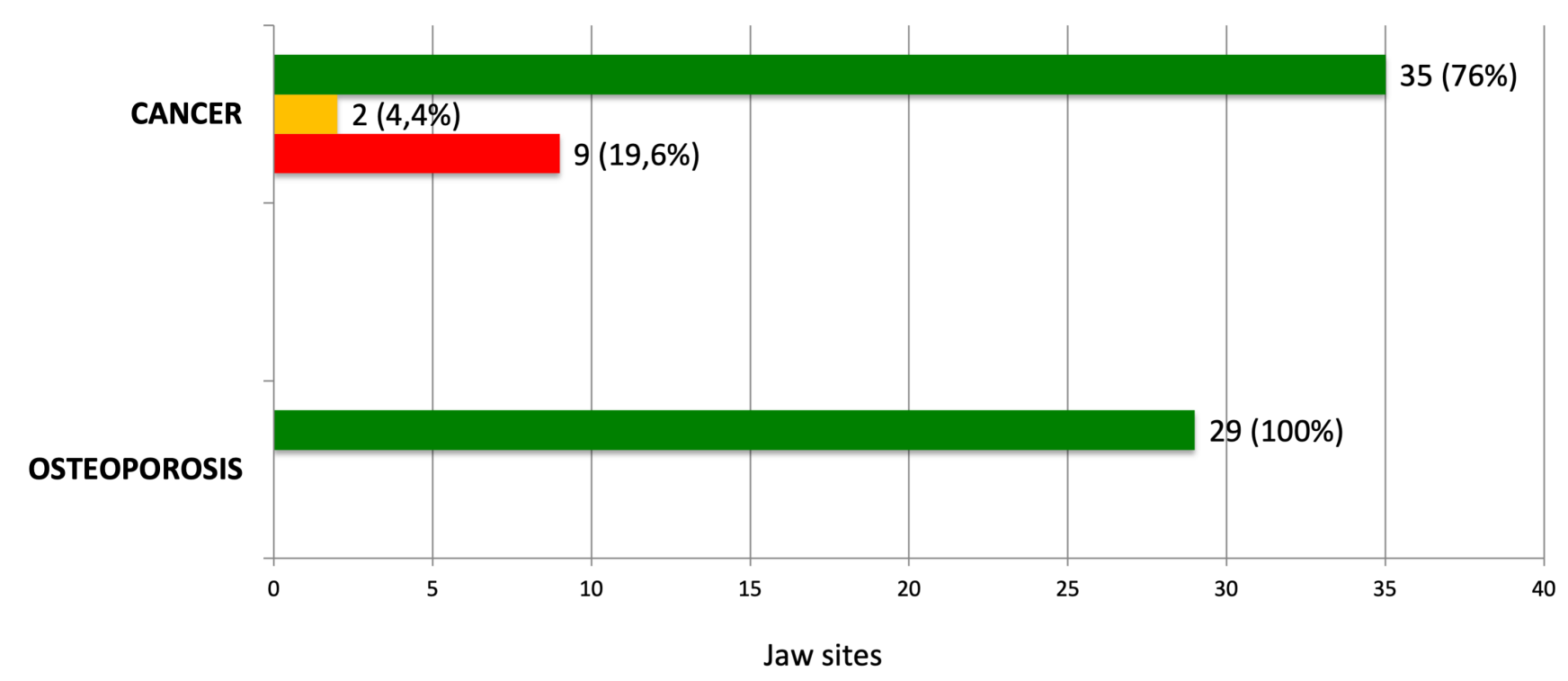

$\square$ HEALING $\square$ SILENT $\square$ RECURRENCE

Graph 3.

When the primary outcome was stratified by the SICMF-SIPMO stage and the type of treatment, bone curettage and sequestrectomy showed the highest CR in stage 1 disease, regardless of the primary disease (88.2\%). Still, reasonable CR can be achieved in MRONJ stage 2 in osteoporosis patients.

As marginal resection is concerned, this surgical approach proved to be successful in stage 2 patients with an overall CR of $81 \%$. Yet, most of the MRONJ stage 2 sites successfully treated with marginal resection pertained to osteoporosis patients $(n=9 / 13)$. Stage 2 MRONJ patients with cancer showed high recurrence rates when treated with marginal resection.

Segmental resection showed high CR in patients with MRONJ stage 2 (88.2\%) and even higher in MRONJ stage 3 (92.9\%) regardless of the primary disease. However, most segmental resections in stage 2 MRONJ were performed in cancer patients (12/15).

All the data are detailed in Graph 4. 


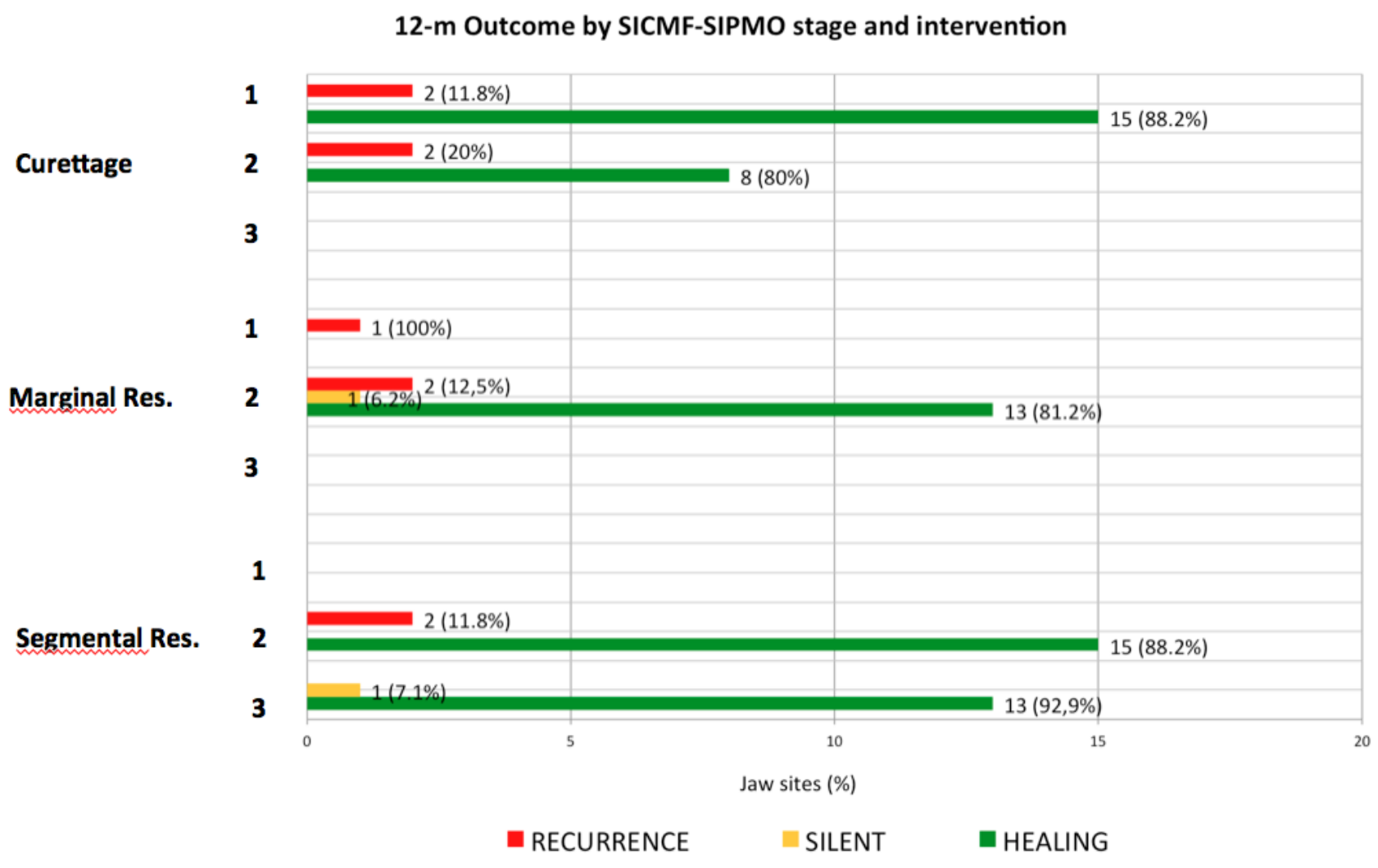

Graph 4.

\section{Discussion and Conclusion}

This is the first study to show the value of the SICMF-SIPMO MRONJ staging system as a predictor of surgical success.

The SICMF-SIPMO treatment protocol is unique in that it assigns patients to specific surgical treatments based on the clinical and radiological extent of jawbone disease. ${ }^{[13]}$ The surgical burden is tuned to the degree of bone destruction so that the early disease stage can be reasonably cured with less aggressive surgery than in advanced stages. It also differentiates the burden of treatment based on the increasing evidence that the clinical course of MRONJ is different for cancer and osteoporosis patients. ${ }^{[12]}$

The present study confirms that surgery can be highly successful in terms of clinical and radiological healing in all disease stages of MRONJ and can be curative in the long term.

We showed that the adoption of the stage-related surgical algorithm proposed by SICMF-SIPMO eliminates the previously reported differences in terms of cure between the maxilla and mandible.

We also verified that bone curettage and sequestrectomy are the best options to treat stage 1 disease, warranting high curative rates with limited morbidity, both for cancer and osteoporosis patients. Though unspecified in the SICMF-SIPMO 
surgical algorithm, bone curettage and sequestrectomy could be also used to treat MRONJ Stage 2 in osteoporosis patients, but not in cancer patients where the likelihood of bone disease recurrence is too high. Nonetheless, marginal resection of MRONJ Stage 2 in osteoporosis patients remains the lead treatment option, while it seems unreasonable to treat with marginal resection MRONJ Stage 2 in cancer patients for the high recurrence rate.

Segmental resection of the jaw remains the most efficient surgical treatment of MRONJ with curative rates that exceed $90 \%$ in more severe cases. Yet, the burden of treatment can be high for patients and necessitates careful selection. This study confirms that segmental resection of the jaw should be limited to cancer patients only in MRONJ Stage 2, while it should be offered to all patients in MRONJ Stage 3, irrespective of the primary disease.

In conclusion, the stage-related surgical algorithm proposed by SICMF-SIPMO can be safely used and it should be implemented to treat MRONJ patients. Further studies are warranted before its final validation.

\section{References}

1. 'Salvatore L. Ruggiero, Thomas B. Dodson, Leon A. Assael, Regina Landesberg, et al. (2009).American Association of Oral and Maxillofacial Surgeons Position Paper on Bisphosphonate-Related Osteonecrosis of the Jaws-2009 Update. Journal of Oral and Maxillofacial Surgery, vol. 67 (5), 2-12. doi:10.1016/j.joms.2009.01.009.

2. 'Salvatore L. Ruggiero, Thomas B. Dodson, John Fantasia, Reginald Goodday, et al. (2014).American Association of Oral and Maxillofacial Surgeons Position Paper on Medication-Related Osteonecrosis of the Jaw-2014 Update. Journal of Oral and Maxillofacial Surgery, vol. 72 (10), 1938-1956. doi:10.1016/j.joms.2014.04.031.

3. 'Morten Schiodt, Sven Otto, Stefano Fedele, Alberto Bedogni, et al. (2019).Workshop of European task force on medication-related osteonecrosis of the jaw-Current challenges. Oral Dis, vol. 25 (7), 1815-1821. doi:10.1111/odi.13160.

4. ^Oliver Ristow, Thomas Rückschloß, Michael Müller, Moritz Berger, et al. (2019).ls the conservative non-surgical management of medication-related osteonecrosis of the jaw an appropriate treatment option for early stages? A longterm single-center cohort study. Journal of Cranio-Maxillofacial Surgery, vol. 47 (3), 491-499. doi:10.1016/j.jcms.2018.12.014.

5. ^R. Fliefel, M. Tröltzsch, J. Kühnisch, M. Ehrenfeld, et al. (2015).Treatment strategies and outcomes of bisphosphonate-related osteonecrosis of the jaw (BRONJ) with characterization of patients: a systematic review. International Journal of Oral and Maxillofacial Surgery, vol. 44 (5), 568-585. doi:10.1016/j.ijom.2015.01.026.

6. ^Alberto Bedogni, Stefano Fedele, Giorgio Bedogni, Matteo Scoletta, et al. (2014).Staging of osteonecrosis of the jaw requires computed tomography for accurate definition of the extent of bony disease. British Journal of Oral and Maxillofacial Surgery, vol. 52 (7), 603-608. doi:10.1016/j.bjoms.2014.04.009.

7. ^L. Bagan, M. Leopoldo-Rodado, R. Poveda-Roda, J. Murillo-Cortes, et al. (2017).Grade of sclerosis in the contralateral mandibular area in osteonecrosis of the jaws. International Journal of Oral and Maxillofacial Surgery, vol. 46 (2), 167-172. doi:10.1016/j.ijom.2016.09.025. 
8. 'Yuxing Guo, Diancan Wang, Yang Wang, Xin Peng, et al. (2016).Imaging features of medicine-related osteonecrosis of the jaws: comparison between panoramic radiography and computed tomography. Oral Surgery, Oral Medicine, Oral Pathology and Oral Radiology, vol. 122 (2), e69-e76. doi:10.1016/j.00oo.2016.04.007.

9. `Max Wilkat, Daman Deep Singh, Isabelle Lutz, Henriette Möllmann, et al. (2020). Use and Evaluation of a ComputerAssisted Examination Method for the Diagnosis and Analysis of Medication-Related Osteonecrosis of the Jaw. Craniomaxillofacial Trauma \& Reconstruction, vol. 14 (1), 36-42. doi:10.1177/1943387520922767.

10. 'Alberto Bedogni, Giorgia Saia, Giordana Bettini, Anita Tronchet, et al. (2011).Long-term outcomes of surgical resection of the jaws in cancer patients with bisphosphonate-related osteonecrosis. Oral Oncology, vol. 47 (5), 420 424. doi:10.1016/j.oraloncology.2011.02.024.

11. ^A Bedogni, V Fusco, A Agrillo, G Campisi. (2012).Learning from experience. Proposal of a refined definition and staging system for bisphosphonate-related osteonecrosis of the jaw (BRONJ). Oral Diseases, vol. 18 (6), 621-623. doi:10.1111/j.1601-0825.2012.01903.x.

12. ${ }^{a, b, c}$ Campisi G, Bedogni A, Fusco V.. (2020). Raccomandazioni clinico-terapeutiche sull'osteonecrosi delle ossa mascellari (ONJ) farmaco-relata e sua prevenzione. Palermo University Press.

13. ${ }^{a, b}$ Giuseppina Campisi, Stefano Fedele, Vittorio Fusco, Giuseppe Pizzo, et al. (2014).Epidemiology. clinical manifestations, risk reduction and treatment strategies of jaw osteonecrosis in cancer patients exposed to antiresorptive agents. Future Oncology, vol. 10 (2), 257-275. doi:10.2217/fon.13.211. 